FORMATION Formation emploi

Revue française de sciences sociales

115 | Juillet-Septembre 2011

Le développement de l'aide à la personne : quelle professionnalisation?

\title{
Introduction : les services d'aide aux personnes
}

Jean-Luc Outin

\section{(2) OpenEdition}

Journals

Édition électronique

URL : http://journals.openedition.org/formationemploi/3408

DOI : $10.4000 /$ formationemploi.3408

ISSN : 2107-0946

Éditeur

La Documentation française

Édition imprimée

Date de publication : 23 septembre 2011

Pagination : 5-7

ISSN : 0759-6340

\section{Référence électronique}

Jean-Luc Outin, «Introduction : les services d'aide aux personnes », Formation emploi [En ligne], 115 |

Juillet-Septembre 2011, mis en ligne le 30 septembre 2011, consulté le 30 octobre 2020. URL : http:// journals.openedition.org/formationemploi/3408; DOI : https://doi.org/10.4000/formationemploi.3408 


\section{DOSSIER}

\section{Introduction}

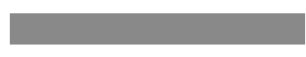

Jean-Luc Outin*

Les services d'aide aux personnes (SAP) connaissent un développement important depuis le début des années 80 . Mais cela n'est pas sans ambiguïté. Sous cette appellation générique, on trouve, en effet, un ensemble de prestations de nature différente, dont le principal point commun est d'être délivré au domicile des personnes aidées.

Ce développement a répondu à différentes préoccupations. Il s'est agi, en particulier, d'apporter des réponses à des besoins croissants engendrés par le vieillissement de la population, une meilleure prise en compte des situations de handicap et la transformation des formes familiales et des conditions d'emploi rendant plus complexe la conciliation entre la vie familiale et la vie professionnelle. Cela a contribué au développement d'interventions sociales nouvelles, combinant notamment celles de l'État, des départements, des organismes de sécurité sociale et des associations pour définir des prestations, dégager des financements et mettre en œuvre des projets variés. Plus récemment, la solvabilisation partielle des besoins a contribué à faire émerger un marché de l'aide aux personnes. D'où l'intérêt des entreprises pour en couvrir certains segments.

De plus, le caractère particulier de ces interventions auprès des personnes et à domicile - fait qu'elles ont été considérées pendant longtemps comme des activités domestiques, requérant des qualités directement transférables depuis la sphère privée où elles s'exerceraient naturellement, sans formation préalable voire sans qualification. D'ailleurs, dès les années 70, les services d'aide ménagère s'étaient ainsi mis en place pour aider les personnes âgées.
Dans le même temps, les transformations des conditions économiques générales ont provoqué des phénomènes d'exclusion du marché du travail. Plus ou moins marqués selon les périodes de haute ou basse conjoncture, ils affectent particulièrement certaines catégories de main-d'œuvre telles que les femmes, les jeunes et les peu qualifiés. Dans ce contexte, les SAP sont apparus comme un gisement d'emplois permettant de lutter contre le chômage, notamment des moins qualifiés.

En réalité, outre leur grande variété et leur spécificité en fonction des populations concernées, ces services aux personnes requièrent des qualités professionnelles multiples dont l'acquisition n'a rien de spontané. Connaissance des dispositifs institutionnels, maitrise de gestes techniques et instauration d'une relation de confiance dans une situation d'aide sont parmi les

* Jean-Luc Outin est économiste, chargé de recherche Cnrs au Centre d'Economie de la Sorbonne (UMR 81-74), directeur du Centre associé Céreq lle de France, membre de l'Observatoire national de la pauvreté et de l'exclusion sociale.

Champ de recherche: les politiques de lutte contre la pauvreté, entre droits sociaux et marché du travail. II a publié récemment : Kerleau M., Outin J.-L. (2010), "Pauvreté et CMU, fragilité des populations et fragilité des droits » in P. Battifoulier, A. Buttard, J-P. Domin (dir), Santé et politiques sociales : entre efficacité et justice, Ed Eska. Outin J.-L (201 1), «Emploi et protection sociale, la redéfinition des liens privilégiés », in M. Lallement (dir) Dictionnaire de l'emploi et de la protection sociale là paraitre). 
principales dimensions à combiner de façon appropriée pour répondre aux besoins de la personne aidée et favoriser son autonomie.

Cette double perspective, réponse à des besoins et gisement d'emplois potentiel, ouvre de nombreuses pistes de réflexion pour l'analyse des services d'aide aux personnes. Parmi elles, ce dossier de Formation Emploi aborde la question de la professionnalisation des emplois et des salariés à partir de trois angles principaux : la formation appréhendée du point de vue de son contenu et de ses modalités de certification ; les activités des aides à domicile caractérisées par leur complexité et leur contenu mouvant ; les acteurs associatifs dont le rôle est traditionnel dans le champ de l'action sociale. Fondées sur des analyses qualitatives, ces approches dressent un tableau contrasté d'un processus à la fois jeune et en plein développement, mais dont les fondements sont sans doute encore incertains. Outre les questions de financement et de redéfinition de l'action publique, l'ambivalence des principes sur lesquels repose l'organisation des activités - l'aide / le service - se reflète dans les difficultés de mise en place des stratégies de professionnalisation. Pour ce secteur, l'enjeu est important. D'abord, parce que la qualité du service, qui repose en large partie sur des qualités de relation et de confiance, ne peut être envisagée indépendamment de la qualité des emplois. De plus, ayant plutôt recruté des personnes parvenues dans la seconde partie de leur vie professionnelle, ce secteur sera confronté prochainement au défi du renouvellement de sa main-d'œuvre ; dès lors, la question se pose de son attractivité et donc des conditions d'emploi et de rémunération des salariés.

Emmanuelle Lada examine la manière dont les modifications de l'action publique contribuent à recomposer le travail d'aide à domicile. Ce mouvement, amorcé depuis une trentaine d'années, a contribué à la reconnaissance de sa fonction sociale. Il s'est fondé et a favorisé l'implication des acteurs pour développer la formation et la qualification des salariés. Cependant, les tendances plus récentes, marquées par la marchandisation de ces services, la mise en concurrence des acteurs qui les organisent et la volonté de maîtriser leurs coûts croissants, définissent un nouveau cadre de régulation. Les temps d'intervention très limités, la parcellisation des tâches et l'isolement accru au travail marquent une nouvelle dynamique de professionnalisation. L'auteur montre que celle-ci s'avère peu propice à l'exercice du travail concret des salariés, fait de réajustements constants pour favoriser le maintien de l'autonomie des personnes.

Emmanuelle Puissant analyse le rôle des associations dans ces processus de professionnalisation des emplois et des personnes, pour en souligner l'ambivalence. Dans un premier temps, l'auteur avance l'idée selon laquelle le rapport domestique au travail qui marque particulièrement les activités d'aide à domicile freine la reconnaissance du travail salarié. Ce trait est accentué par les caractéristiques propres de l'acteur associatif marqué par la place du travail bénévole et l'importance de la relation avec l'usager ; de plus, l'émiettement associatif favorise le maintien, à un niveau interpersonnel, des tensions nées dans le cadre du travail professionnel. Dans un second temps, les avantages du cadre associatif pour la création d'un collectif favorable à la professionnalisation sont soulignés, notamment par rapport aux situations d'emploi direct largement prépondérantes dans le secteur de l'aide à domicile. Outre l'expression des conflits entourant les relations de travail, cela contribue à l'apparition de fonctions d'encadrement intermédiaire propices à l'émergence d'un espace collectif de travail ; celui-ci étant susceptible d'enclencher ce que l'auteur dénomme « un cercle vertueux de la professionnalisation ».

Gérald Houdeville et Laurent Riot s'interrogent sur ce que produisent les formations dispensées pour acquérir les dispositions nécessaires à un agir professionnel approprié. Ils en soulignent le double aspect moral et technique. Ils rappellent les principales caractéristiques des publics des formations et l'organisation du système de formation professionnelle. Ils montrent ainsi que les connaissances dispensées visent l'apprentissage d'un vocabulaire spécifique pour désigner les problèmes des personnes à accompagner, d'une part, et la connaissance du cadre institutionnel et légal qui organise l'intervention publique dans ce domaine, d'autre part. La professionnalisation passe par l'acquisition d'une distance indispensable avec la personne aidée. La formation vise ainsi à développer des dispositions relationnelles fondées sur la maîtrise de différents modèles plus ou moins standardisés. L'importance de la dimension relationnelle n'exclut pas, cependant, la maîtrise des actes 
techniques ; d'ailleurs, l'opposition entre ces deux pôles est sans doute vaine, dans la mesure où c'est l'interaction entre les deux dimensions qui, pour une large part, caractérise un professionnalisme maîtrisé impliquant de grandes capacités d'adaptation. Cela se traduit par la combinaison entre les apports théoriques et les échanges d'expérience en cours de formation, conférant une place particulière aux stagiaires.

Ghislaine Doniol-Shaw s'intéresse également aux formations pour souligner le paradoxe entre le besoin reconnu d'une main-d'œuvre qualifiée et l'absence de filière susceptible d'y répondre. Au-delà des activités qu'il implique, le travail d'aide aux personnes est bien spécifique puisqu'il doit être redéfini en permanence en fonction de l'état des bénéficiaires, et notamment de leur degré d'autonomie. La démarche de « faire avec » et non de « faire pour » inscrit la professionnalisation dans le registre de l'aide et non du service. Dès lors, elle conduit à mettre l'accent sur la spécificité du métier d'aide aux personnes âgées qui s'est éloignée à la fois d'une fonction d'aide-ménagère et de l'intervention auprès de jeunes enfants. Si les formations sont nombreuses, les diplômes sont quasiment tous de premier niveau; or, dans la branche professionnelle de l'aide à domicile, il existe une correspondance assez stricte entre le classement des emplois et la détention d'un diplôme. Cela nuit à la carrière des personnes qui ont une expérience non sanctionnée par un diplôme. De même, l'absence de diplôme spécifique au métier de l'aide aux personnes âgées entrave la validation des acquis professionnels pour les salarié(e)s qui ne maîtrisent pas les connaissances requises par l'intervention en direction d'autres publics. Dans ces conditions, l'auteur milite pour un changement du cadre de l'organisation de la formation qui combine mieux les deux dimensions d'aide et de soin. 